УДК 538.9:536.6

\title{
СТРУКТУРООБРАЗОВАНИЕ ПОЛИМЕРНЫХ МИКРО- И НАНОКОМПОЗИТОВ НА ОСНОВЕ ПОЛИКАРБОНАТА В ПРОЦЕССАХ ИХ КРИСТАЛЛИЗАЦИИ
}

\author{
Долинский А.А. ${ }^{1}$, академик НАН Украины, Фиалко Н.М. ${ }^{1}$, член-корреспондент НАН Украины, \\ Динжос P.B. ${ }^{2}$, канд. физ.-мат. наук, Навродская Р.А. ${ }^{1}$, канд. техн. наук \\ ${ }^{1}$ Институт технической теплофизики НАН Украинь,, ул. Желябова, 2 а, Киев, 03680, Украина \\ ${ }^{2}$ Николаевский национальный университет им. В.А.Сухомлинского, ул. Никольская, 24, Николаев, \\ 540030, Украина
}

Наведено дані експериментальнотеоретичних досліджень щодо механізмів структуроутворення при кристалізації полімерних композитів на основі полікарбонату, наповнених вуглецевими нанотрубками або мікрочастинками алюмінію. Представлено результати аналізу одержаних експериментальних даних 3 кінетики кристалізації у відповідності з рівняннями нуклеації та Колмогорова-Аврамі. Встановлено механізми кристалізації розглянутих полімерних мікро- і нанокомпозитів на початковій стадії кристалізації (стадіï нуклеації) та стадії кристалізації в об'ємі матеріалу в цілому.
Приведены данные экспериментально-теоретических исследований механизмов структурообразования при кристаллизации полимерных композитов на основе поликарбоната, наполненных углеродными нанотрубками или микрочастицами алюминия. Представлены результаты анализа полученных экспериментальных данных по кинетике кристаллизации в соответствии с уравнениями нуклеации и Колмогорова-Аврами. Установлены механизмы кристаллизации рассмотренных полимерных микро- и нанокомпозитов на начальной стадии кристаллизации (стадии нуклеации) и стадии кристаллизации в объеме материала в целом.
The data of experimental and theoretical studies of the mechanisms of structure formation in the crystallization of polymer composites based on polycarbonate filled with carbon nanotubes or microparticles of aluminum are given. The results of the analysis of the experimental data on the crystallization kinetics in accordance with the equations of nucleation and Kolmogorov-Avrami are submitted. The mechanisms of crystallization of considered polymeric micro- and nanocomposites on the initial crystallization step (nucleation step) and the step of crystallization in the volume of material as a whole are established.

Библ. 8 , табл. 4, рис. 3.

Ключевые слова: полимерные микро- и нанокомпозиты, механизмы структурообразования, кинетика кристаллизации.

$a_{m}$ - приведенный параметр нуклеации;

$\Delta E-$ энергия активации;

$f$ - относительная доля механизма кристаллиза-

ции, связанного с кристаллизацией собственно полимерной матрицы;

$\Delta H_{m}^{o}$ - энтальпия плавления;

$k$ - постоянная Больцмана;

$K_{m}$ - приведенный транспортный барьер;

$K_{n}^{m}$ - эффективная константа скорости;

$m$ - безразмерный параметр формы;

$n$ - псевдопараметр формы;

$Q, Q_{\max }$ - текущее и максимальное значение теплового потока;

$R$ - коэффициент корреляции;

$T$ - температура;

$T_{N}, T_{K}$ - температура начала и окончания кристаллизации;
$\Delta T$ - температурный интервал кристаллиза-ции;

$T_{M}$ - температура расплава, отвечающая максимальному значению теплового потока;

$t$ - время;

$V_{t}$ - скорость охлаждения;

$\mathrm{Z}$ - энергия нуклеации;

$\alpha$ - относительная объемная доля кристаллической фазы;

$\tau-$ приведенное время, $\tau=V_{t} \cdot t$;

$\chi^{2}$ - дисперсия;

$\omega$ - массовая доля наполнителя.

\section{Введение}

В последний период наблюдается тенденция к расширению областей использования полимерных материалов за счет применения их различных композиций. Данные композиции, как 
известно, обладают существенно более широким спектром физико-механических и технологических свойств. При этом особо выделяются полимерные микро- и нанокомпозиты, свойства которых улучшены введением в полимерную матрицу относительно незначительного количества частиц наполнителя соответствующих размеров [1-5].

Задачи разработки новых полимерных микро- и нанокомпозитов требуют проведения углубленных исследований, направленных на изучение особенностей формирования их структуры в процессе кристаллизации, что в большой мере определяет свойства получаемых материалов.

Данная статья посвящена экспериментальнотеоретическим исследованиям закономерностей структурообразования полимерных микро- и нанокомпозитов на основе поликар-боната, наполненных углеродными нанотрубками или микрочастицами алюминия.

\section{Постановка задачи и методика проведения исследований}

В задачу исследования входило установление механизмов структурообразования при охлаждении полимерных композитов из расплава. При этом рассмотрению подлежали данные механизмы на стадии зарождения отдельных структурно упорядоченных подобластей (стадия нуклеации) и на стадии образования таких подобластей во всем объеме композита (стадия кристаллизации в объеме материала в целом). Исследования проводились для указанных выше полимерных композитов при изменении массовой доли наполнителей от 0,2 до 4 \% и варьировании скорости охлаждения композита от $0,0083 \mathrm{~K} / \mathrm{c}(0,5 \mathrm{~K} / \mathrm{мин})$ до $0,333 \mathrm{~K} / \mathrm{c}(20 \mathrm{~K} /$ мин $)$.

В работе ставилась также задача проведения сравнительного анализа механизмов структурообразования в рассматриваемых композитах и собственно полимерной матрице.

Используемая экспериментально-теоретическая методика установления механизмов структурообразования включала два этапа, первый из которых состоял в экспериментальном определении экзотерм затвердевания композита при его охлаждении из расплава с заданной постоянной скоростью. Здесь, тепловой поток, отводимый от композита, определялся в сухой атмосфере азота методом дифференциальной сканирующей калориметрии (ДСК) на установке Perkin Elmer DSC-2 с модифицированным программным обеспечением от IFA Gmb, Ulm [6]. При этом образец, помещенный в ячейку, нагревался до температуры 550 К, превышающей температуру плавления матрицы примерно на 50 К, выдерживался при данной температуре в течение 180 с и далее охлаждался до температуры равной 400 К при фиксированной скорости его охлаждения.

Второй этап применяемой методики заключался в теоретическом определении на основе полученных экспериментальных данных характеристик структурообразования на стадии начала формирования в материале отдельных упорядоченных структур с использованием уравнения нуклеации и в нахождении параметров образования таких структур на стадии их формирования во всем объеме композита с применением уравнения Колмогорова-Аврами.

\section{Результаты исследований и их анализ}

На рис. 1, 2 и в табл. 1, 2 приведены характерные результаты экспериментальных исследований, касающиеся определения экзотерм затвердевания для рассматриваемых полимерных микро- и нанокомпозитов.

Рассмотрим в начале особенности влияния скорости охлаждения композитов $V_{t}$ на основные параметры процесса их кристаллизации. Согласно полученным данным для обоих рассматриваемых композитов при разной массовой доле наполнителя имеет место в целом аналогичный характер изменения этих параметров с ростом величины $V_{t}$ (рис. 1,2 ). Здесь в первую очередь обращает на себя внимание весьма существенное снижение максимума отводимого от композита теплового потока $Q_{\max }$ с повышением скорости охлаждения при заметном смещении положения данного.максимума.на кривой $Q=f(T)$ в область более низких темератур. Так, для микрокомпозита, содержащего $0,2 \%$ алюминия, величина $Q_{\max }$ $=1,84$ мВт при $V_{t}=0,0083 \mathrm{~K} /$ с и снижается до 1,02 мВт при $V_{t}=0,333 \mathrm{~K} / \mathrm{c}$. Что же касается температуры $T_{\mathrm{M}}$, отвечающей тепловому потоку $Q_{\text {max }}$, то для указанных скоростей $V_{t}$ она равна $463,5 \kappa$ и 448,9 К соответственно.

Повышение скорости охлаждения композита $V_{t}$ приводит также к снижению температур начала 
$T_{N}$ и конца $T_{K}$ кристаллизации. При этом заметно повышается интервал температур кристализации $\Delta T\left(\Delta T=T_{N}-T_{K}\right)$. Например, для нанокомпозита, содержащего 4 \% углеродных нанотрубок, указанный интервал $\Delta T$ составляет $6,5 \mathrm{~K}$ при $V_{t}=$ $=0,0083 \mathrm{~K} /$ с и достигает 18,9 К при $V_{t}=0,333 \mathrm{~K} / \mathrm{c}$.

Остановимся вкратце на рассмотрении эффектов влияния массовой доли наполнителя $\omega$ на характер полученных экзотерм кристаллизации. Как видно из рис. 1 и 2, с повышением $\omega$ наблюдается трансформация унимодального пика на кривой $Q=f(T)$ в бимодальный. При этом указанная бимодальность с ростом $\omega$ становится

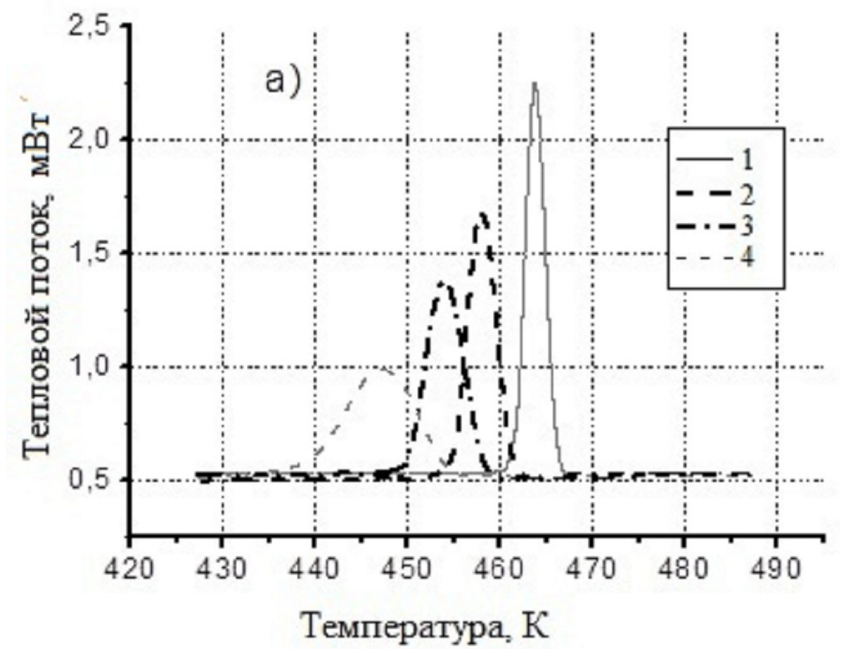

все более ярко выраженной. Важно подчеркнуть, что массовая доля наполнителя $\omega$, при которой обнаруживается отмеченная трансформация, оказывается различной для разных композиционных материалов. Как следует из результатов выполненных экспериментальных исследований, в случае полимерного композита, наполненного микрочастицами алюминия, бимодальный пик на кривых $Q=f(T)$ появляется при значении $\omega$, равном $1 \%$, в случае же нанокомпозита, наполненного углеродными нанотрубками, он обнаруживается при $\omega=0,5 \%$.

При проведении экспериментов были полу-

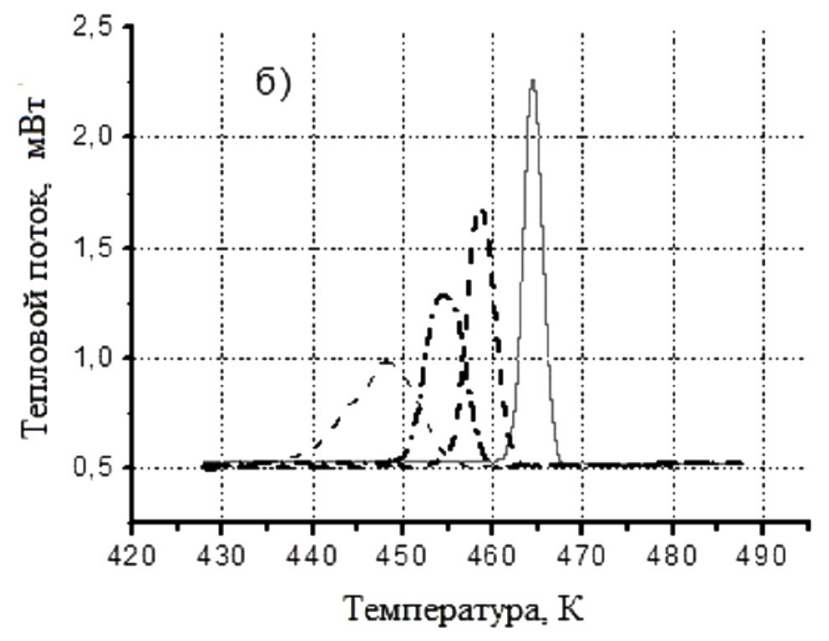

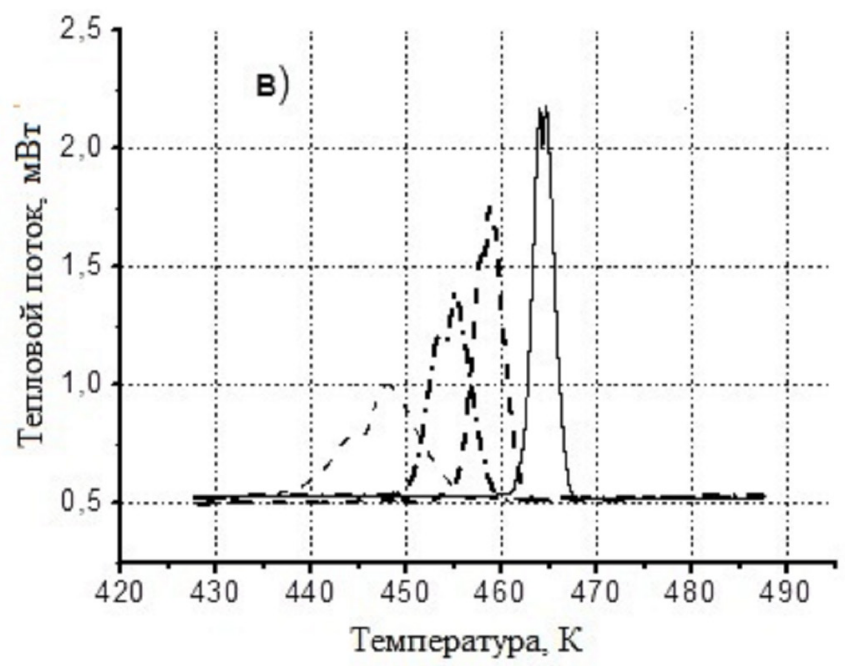

Рис. 1. Экзотермы кристаллизации для полимерных нанокомпозитов на основе поликарбоната, наполненных углеродными нанотрубками, при содержании наполнителей $\omega=0,2 \%$ (a), 0,3 \% (б) и 4,0 \% (в) при разных скоростях охлажсдения композита из расплава: $1-V_{t}=0,0083 K / c ; 2-0,0333 K / c ; 3-0,0833 K / c ; 4-0,333 K / c$. 

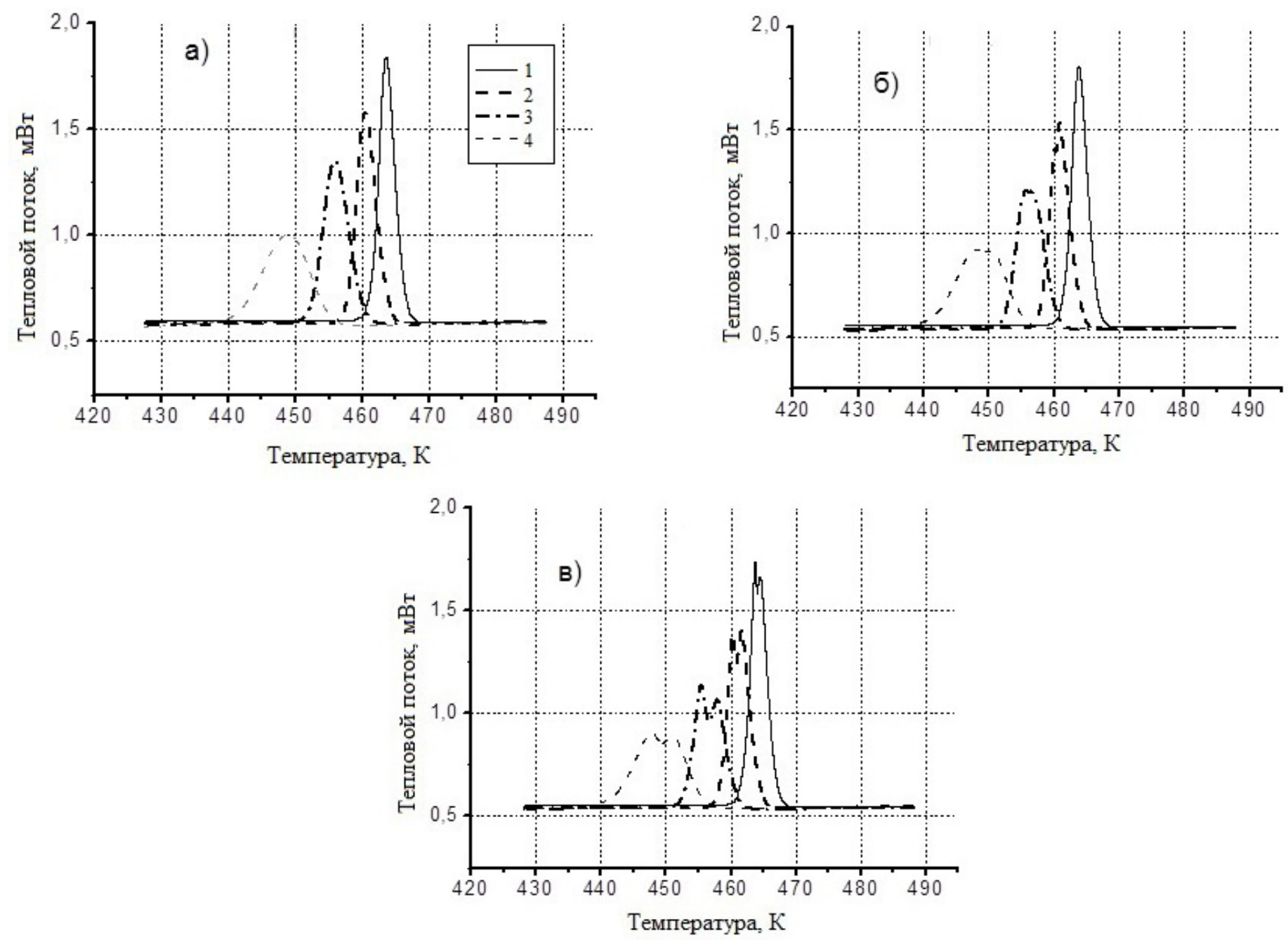

Рис. 2. Экзотермы кристаллизации для полимерных композиционных материалов на основе поликарбоната, наполненных микрочастицами алюминия, при содержании наполнителя $\omega=0,2 \%$ (a), 1,0 (б) и 4,0\% (в) при различных скоростях охлажндения композита из расплава: $1-V_{t}=0,0083 \mathrm{~K} / \mathrm{c} ; 2-0,0333 \mathrm{~K} / \mathrm{c} ; 3-0,0833 \mathrm{~K} / \mathrm{c} ; 4-0,333 \mathrm{~K} / \mathrm{c}$.

чены также соответствующие экзотермы кристаллизации для полимерной матрицы при различных скоростях $V_{t}$.охлаждения из расплава. Здесь характер экзотерм в целом аналогичен таковым для рассматриваемых композитов при относительно небольшом содержании наполнителя $(\omega=0,2 \%)$. То есть при всех исследованных скоростях охлаждения $V$ экзотермы кристаллизации полимерной матрицы содержали лишь унимодальные пики, уровень которых снижался с ростом $V_{t}$.

Полученные экспериментальные данные о кинетике кристаллизации рассматриваемых композитов, как уже отмечалось, служили основой для теоретического определения соответствую- щих параметров структурообразования.

Рассмотрим особенности структурообразования изучаемых композитов на начальной стадии их кристаллизации - стадии нуклеации. Здесь определению подлежали такие основные характеристики кристаллообразования, как приведенный параметр нуклеации $a_{m}$ и приведенный транспортный барьер $K_{m}$ $a_{m}=Z / k\left(\Delta H_{m}^{o}\right)^{m}, K_{m}=A \exp \left(\Delta E / k T_{N}\right)$.

Кроме того, необходимым являлось проведение анализа в части размерности кристаллообразования, связанной с параметром $m$.

Для нахождения величины $a_{m}$ и $K_{m}$ использо- 
валось уравнение нуклеации [7]

$$
\begin{aligned}
& \ln \left\{V_{t}\left[(m+1) T_{N}-T_{\mathrm{M}}\right]\left(T_{\mathrm{M}}\right)^{m} / T_{N}^{2}(\Delta T)^{m+1}\right\}= \\
& =\ln \left(K_{m} / a_{m}\right)-a_{m}\left(T_{\mathrm{M}}\right)^{m} / T_{N}(\Delta T)^{m} .
\end{aligned}
$$

Решение данного уравнение осуществлялось с применением метода наименьших квадратов и использованием полученных в результате экспериментальных исследований величин $T_{N}, T_{M}$ и $\Delta T$. При этом в целях анализа размерности кристаллообразования уравнения (1) решалось для $m=1$ и $m=2$.

В табл. 3 представлены результаты решения уравнения (1) для композитов на основе поликарбоната, наполненных углеродными нанотрубками либо микрочастицами алюминия, при различном содержании наполнителя и разных значениях параметра $m$. Как видно из табл. 3 , при $m=1$ и $m=2$ корреляция между экспериментальными и расчетными данными вполне удовлетворительна. При этом, однако, в случае $m=1$ в исследуемом диапазоне изменения параметров значения коэффициентов корреляции $R_{1}$ оказываются существенно меньшими, чем значения соответствующих коэффициентов $R_{2}$ при $m=2$. Таким образом, приведенные данные свидетельствуют о наличии двух механизмов структурообразования на начальной стадии кристаллизации рассматриваемых полимерных композитов, а именно, двумерного плоскостного $(m=1)$ и трехмерного, объемного $(m=2)$. Отмеченное заметное превышение величины $R_{2}$ над $R_{1}$ указывает на некоторое преобладание объемного механизма над плоскостным.

Что касается изменения с ростом содержания наполнителя $\omega$, приведенного параметра нуклеации $a_{m}$ и приведенного транспортного барьера $K_{m}$, то для рассматриваемых композитов характер данного изменения оказывается аналогичным при $m=1$ и $m=2$. С повышением $\omega$ значения приведенного параметра нуклеации $a_{m}$ увеличиваются, а транспортного барьера $K_{m}^{m}-$ падают (см. данные табл. 3). Рост $a_{m}$ связан, как известно, с увеличением скорости кристаллизации

Табл. 1. Характеристики процесса кристаллизации полимерного нанокомпозита на основе поликарбоната, наполненного углеродными нанотрубками, при разном содержании наполнителя $\omega$ и различ-

\begin{tabular}{|c|c|c|c|c|c|}
\hline$\omega, \%$ & $T_{N}, K$ & $T_{M}, K$ & $T_{k}, K$ & $\Delta T, K$ & $Q_{\max }, M B m$ \\
\hline \multicolumn{6}{|c|}{$V_{t}=0,0083 \mathrm{~K} / \mathrm{c}$} \\
\hline 0 & 467,5 & 463,0 & 460,0 & 7,5 & 1,79 \\
\hline 0,2 & 466,9 & 463,7 & 461,0 & 5,9 & 2,24 \\
\hline 0,3 & 467,6 & 464,3 & 461,2 & 6,4 & 2,27 \\
\hline 4 & 467,4 & 464,$0 ; 464,6$ & 460,9 & 6,5 & 2,18 \\
\hline \multicolumn{6}{|c|}{$V_{t}=0,0166 \mathrm{~K} / \mathrm{c}$} \\
\hline 0 & 467,1 & 462,3 & 459,2 & 7,9 & 1,70 \\
\hline 0,2 & 465,0 & 460,8 & 457,8 & 7,2 & 1,94 \\
\hline 0,3 & 465,6 & 461,5 & 457,8 & 7,8 & 1,95 \\
\hline 4 & 465,6 & 461,$7 ; 461,0$ & 458,0 & 7,6 & 1,90 \\
\hline \multicolumn{6}{|c|}{$V_{t}=0,0333 \mathrm{~K} / \mathrm{c}$} \\
\hline 0 & 465,2 & 460,0 & 457,0 & 8,2 & 1,55 \\
\hline 0,2 & 462,4 & 458,0 & 454,2 & 8,2 & 1,68 \\
\hline 0,3 & 463,0 & 458,6 & 454,4 & 8,6 & 1,70 \\
\hline 4 & 462,9 & 458,$7 ; 457,8$ & 454,6 & 8,3 & 1,73 \\
\hline \multicolumn{6}{|c|}{$V_{t}=0,0833 \mathrm{~K} / \mathrm{c}$} \\
\hline 0 & 461,2 & 455,4 & 451,9 & 9,3 & 1,32 \\
\hline
\end{tabular}
ных скоростях Vt охлаждения композита из расплава 


\begin{tabular}{|c|c|c|c|c|c|}
\hline 0,2 & 458,2 & 453,7 & 449,7 & 8,5 & 1,37 \\
\hline 0,3 & 458,7 & 454,5 & 449,8 & 8,9 & 1,28 \\
\hline 4 & 458,8 & 455,$1 ; 453,4$ & 450,2 & 8,6 & 1,38 \\
\hline \multicolumn{6}{|c|}{$V_{+}=0,166 \mathrm{~K} / \mathrm{c}$} \\
\hline 0 & 457,9 & 451,2 & 446,9 & 11,0 & 1,16 \\
\hline 0,2 & 457,5 & 451,2 & 445,1 & 12,4 & 1,19 \\
\hline 0,3 & 458,1 & 451,$9 ; 450,1$ & 445,5 & 12,6 & 1,20 \\
\hline 4 & 458,1 & 452,$1 ; 450,1$ & 445,2 & 12,9 & 1,21 \\
\hline \multicolumn{7}{|c|}{$V_{+}=0,333 \mathrm{~K} / \mathrm{c}$} \\
\hline 0 & 456,5 & 448,4 & 441,5 & 15,0 & 0,97 \\
\hline 0,2 & 455,0 & 446,7 & 438,3 & 16,7 & 0,98 \\
\hline 0,3 & 455,6 & 448,$0 ; 444,0$ & 437,7 & 17,9 & 0,99 \\
\hline 4 & 455,6 & 448,$1 ; 443,9$ & 436,7 & 18,9 & 1,00 \\
\hline
\end{tabular}

на ее начальной стадии, а снижение $K_{m}-$ с уменьшением ограничений по транспорту сегментов матрицы через поверхность ламель-кристалл.

Важно также подчеркнуть, что согласно результатам выполненных экспериментальных исследований параметр нуклеации $a_{m}$ и транспортный барьер $K_{m}$ претерпевают весьма резкое изменение в области относительно низких значений содержания наполнителя $\omega$, а именно, при изменении $\omega$ от 0,2 до 0,5 \% для углеродных нанотрубок и от 0,2 до 1,0 \% - для микрочастиц алюминия. Дальнейшее повышение содержания наполнителя оказывает существенно меньшее влияние на указанные параметры. Такой характер поведения величин ат и $K_{m}$ связан, по-видимому, с прохождением при повышении $\omega$ первого порога перколяции, отвечающего образованию непрерывного перколяционного кластера [8].

Перейдем далее к рассмотрению закономерностей структурообразования исследуемых полимерных композитов на второй стадии кристаллизации - кристаллизация в объеме материала в целом. Одной из наиболее важных характеристик структурообразования на данной стадии является псевдопараметр формы n, связанный с механизмом кристаллизации в плане его пространственных особенностей. Последний определялся посредством анализа полученных экспериментальных данных в рамках стандартного уравнения Колмогорова-Аврами [7] $\alpha(\tau)=1-\exp \left(-K_{n} \tau^{n}\right)$,

где $\alpha(\tau)$ представляет собой отношение текущего и конечного объемов закристаллизовавшегося материала.

Выполненный анализ показал, что при таком подходе расхождение экспериментальных и расчетных данных оказываются весьма существенными (см. значения дисперсии $\chi^{2}$ для уравнения (2) в табл. 4 и соответствующие данные на рис. 3). Данное обстоятельство обусловило необходимость рассмотрения возможности интерпретации экспериментальных данных в предположении наличия двух механизмов кристаллообразования, первый из которых связан с кристаллизацией собственно полимерной матрицы (реализующейся на флуктуациях плотности полимера), и второй - с кристаллизацией, в которой роль ее центров выполняют частицы наполнителя. Согласно этому полученные результаты экспериментальных исследований анализировались в соответствии с модифицированным уравнением Колмогорова-Аврами [7]

$\alpha(\tau)=f\left[1-\exp \left(-K_{n}^{\prime} \tau^{n^{\prime}}\right)\right]+(1-f) \cdot\left[1-\exp \left(-K_{n}^{\prime \prime} \tau^{n^{\prime \prime}}\right)\right]$,

здесь надстрочные индексы “ ' " и “ " " относят величины к первому и второму из указанных механизмов.

Полученные результаты свидетельствуют о 
Табл. 2. Характеристики процесса кристаллизации полимерного микрокомпозита, наполненного микрочастицами алюминия, при разном содержании наполнителя $\omega$ и различных скоростях $V_{t}$ охлаждения композита из расплава

\begin{tabular}{|c|c|c|c|c|c|}
\hline$\omega, \%$ & $T_{N}, K$ & $T_{M}, K$ & $T_{k}, K$ & $\Delta T, K$ & $Q_{\max }, M B m$ \\
\hline \multicolumn{6}{|c|}{$V_{t}=0,0083 \mathrm{~K} / \mathrm{c}$} \\
\hline 0 & 467,5 & 463,0 & 460,0 & 7,5 & 1,79 \\
\hline 0,2 & 468,0 & 463,5 & 460,6 & 7,4 & 1,84 \\
\hline 1 & 468,7 & 463,8 & 460,5 & 8,2 & 1,81 \\
\hline 4 & 468,5 & 464,$4 ; 463,7$ & 460,8 & 7,7 & 1,75 \\
\hline \multicolumn{6}{|c|}{$V_{t}=0,0166 \mathrm{~K} / \mathrm{c}$} \\
\hline 0 & 467,1 & 462,3 & 459,2 & 7,9 & 1,70 \\
\hline 0,2 & 467,6 & 462,8 & 459,7 & 7,9 & 1,75 \\
\hline 1 & 468,3 & 463,$3 ; 462,6$ & 459,6 & 8,7 & 1,71 \\
\hline 4 & 468,1 & 463,$7 ; 462,8$ & 459,5 & 8,6 & 1,66 \\
\hline \multicolumn{6}{|c|}{$V_{t}=0,0333 \mathrm{~K} / \mathrm{c}$} \\
\hline 0 & 465,2 & 460,0 & 457,0 & 8,2 & 1,55 \\
\hline 0,2 & 465,7 & 460,5 & 457,6 & 8,1 & 1,59 \\
\hline 1 & 466,3 & 460,$8 ; 460,0$ & 457,5 & 8,8 & 1,57 \\
\hline 4 & 466,2 & 461,$7 ; 460,4$ & 457,8 & 8,4 & 1,41 \\
\hline \multicolumn{6}{|c|}{$V_{+}=0,0833 \mathrm{~K} / \mathrm{c}$} \\
\hline 0 & 461,2 & 455,4 & 451,9 & 9,3 & 1,32 \\
\hline 0,2 & 461,7 & 455,9 & 452,3 & 9,4 & 1,38 \\
\hline 1 & 462,4 & 456,$9 ; 455,5$ & 451,9 & 10,5 & 1,22 \\
\hline 4 & 462,2 & 458,$0 ; 455,5$ & 452,1 & 10,1 & 1,14 \\
\hline \multicolumn{6}{|c|}{$V_{t}=0,166 \mathrm{~K} / \mathrm{c}$} \\
\hline 0 & 457,9 & 451,2 & 446,9 & 11,0 & 1,16 \\
\hline 0,2 & 458,4 & 451,7 & 447,4 & 11,0 & 1,20 \\
\hline 1 & 459,1 & 453,$6 ; 451,5$ & 446,8 & 12,3 & 1,10 \\
\hline 4 & 459,0 & 453,$9 ; 451,7$ & 447,0 & 12,0 & 1,09 \\
\hline \multicolumn{6}{|c|}{$V_{t}=0,333 \mathrm{~K} / \mathrm{c}$} \\
\hline 0 & 456,5 & 448,4 & 441,5 & 15,0 & 0,97 \\
\hline 0,2 & 457,0 & 448,9 & 440,6 & 16,4 & 1,02 \\
\hline 1 & 457,7 & 450,$9 ; 447,5$ & 440,4 & 17,3 & 0,94 \\
\hline 4 & 457,4 & 451,$0 ; 447,8$ & 440,2 & 17,2 & 0,90 \\
\hline
\end{tabular}

том, что для поликарбоната значения псевдопараметра формы $n \approx 4$. Последнее означает, что кристаллизация происходит по так называемому механизму напряженной матрицы.

Что касается второго из указанных механиз- мов, то согласно полученным данным он существенно зависит от массовой доли наполнителя $\omega$. Так, для нанокомпозита, наполненного углеродными нанотрубками, при $\omega=0,2$ \% псевдопараметр формы $n \approx 4$. То есть здесь реализуется 
Табл. 3. Параметры структурообразования на начальной стадии кристаллизации полимерных композитов на основе поликарбоната при различном содержании наполнителей $\omega$

\begin{tabular}{|c|c|c|c|c|c|c|}
\hline$\omega, \%$ & $a_{1}, K$ & $K_{1}, 1 / \mathrm{c}$ & $R_{1}$ & $a_{2}, 10^{-6} \mathrm{~K}$ & $K_{2}, 1 / \mathrm{c}$ & $R_{2}$ \\
\hline \multicolumn{7}{|c|}{ Наполнитель - углеродные нанотрубки } \\
\hline 0 & 0,325 & 0,056 & 0,8142 & 1,82 & 0,940 & 0,9997 \\
\hline 0,2 & 0,329 & 0,054 & 0,8551 & 1,86 & 0,910 & 0,9949 \\
\hline 0,3 & 0,356 & 0,025 & 0,8964 & 2,13 & 0,470 & 0,9945 \\
\hline 4 & 0,362 & 0,022 & 0,8821 & 2,18 & 0,450 & 0,9946 \\
\hline \multicolumn{7}{|c|}{ Наполнитель- микрочастицы алюминия } \\
\hline 0 & 0,325 & 0,056 & 0,8142 & 1,82 & 0,940 & 0,9997 \\
\hline 0,2 & 0,327 & 0,052 & 0,8255 & 1,84 & 0,930 & 0,9964 \\
\hline 1 & 0,345 & 0,039 & 0,8412 & 1,95 & 0,530 & 0,9962 \\
\hline 4 & 0,351 & 0,037 & 0,8315 & 1,99 & 0,500 & 0,9975 \\
\hline
\end{tabular}
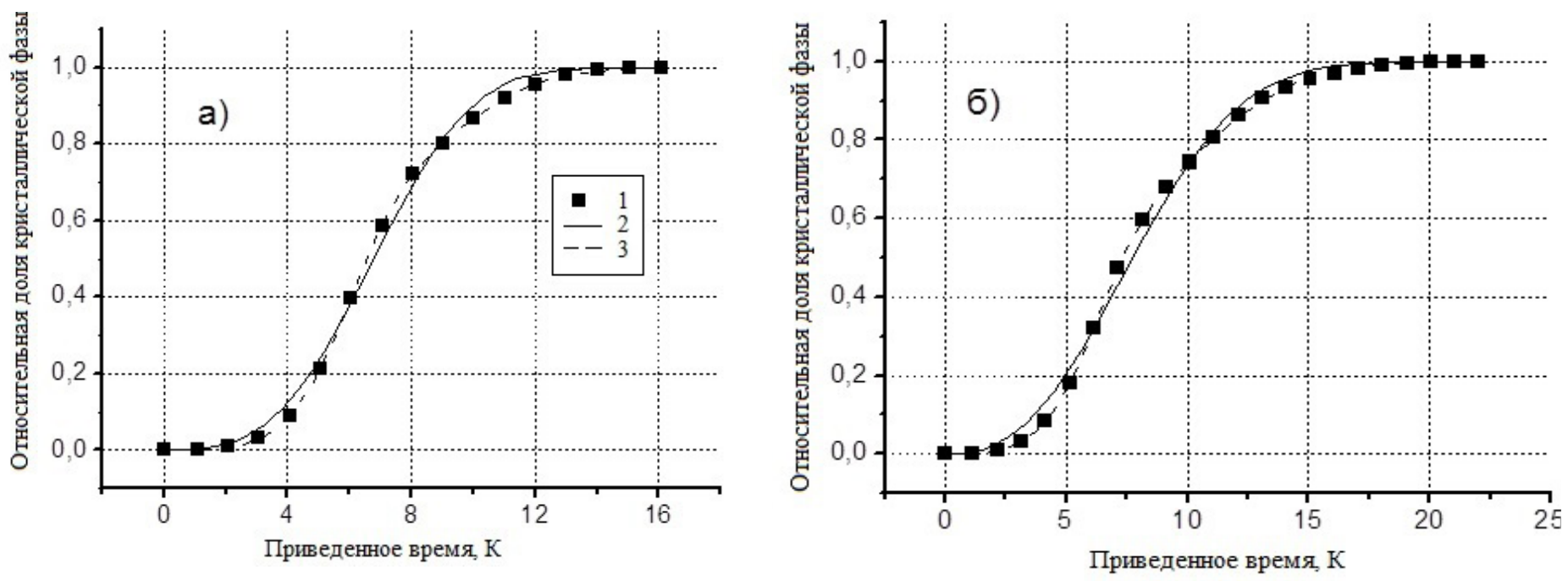

Рис. 3. Зависимость относительной объемной доли кристаллической фазы $\alpha(\tau)$ от приведенного времен $\tau$ для полимерного композита, наполняемого углеродными нанотрубками (a) и микрочастицами алюминия (б) при $\omega=4 \%$ и $V_{t}=0,0166 \mathrm{~K} / \mathrm{c}$; 1 -результаты эксперимента; 2, 3 - данные, отвечающие уравнениям (2) и (3).

механизм аналогичный механизму кристаллизации полимерной матрицы. Повышение массовой доли углеродных нанотрубок до 0,3 \% приводит к тому, что указанный второй механизм становится поверхностным $(n \approx 2)$. При этом данный механизм сохраняется при дальнейшем повышении $\omega$.

Несколько иная картина наблюдается для полимерных микрокомпозитов, наполненных частицами алюминия. В этом случае при $\omega=0,2 \%$ механизм кристаллизации остается таким же как и в полимерной матрице. При $\omega=1,0 \%$ происходит трансформация данного механизма в одномерный $(n \approx 1)$, при котором имеет место образование углеродных игольчатых кристаллических структур. Дальнейшее повышение $\omega$ не приводит к изменению указанного одномерного механизма. 
Табл. 4. Параметры структурообразования на стадии кристаллизации в объеме полимерных композитов на основе поликарбоната при различной доле наполнителей $\omega$ и разной скорости охлаждения $V_{t}$

\begin{tabular}{|c|c|c|c|c|c|c|c|c|}
\hline \multirow{2}{*}{$\begin{array}{c}V_{t}, \\
\mathrm{~K} / \mathrm{c}\end{array}$} & \multicolumn{3}{|c|}{ Уравнение (2) } & \multicolumn{5}{|c|}{ Уравнение (3) } \\
\hline & $n$ & $K_{n}, 10^{-5} K^{-n}$ & $\chi^{2}, 10^{-5}$ & $f$ & $\mathrm{~K}_{n}^{\prime}, 10^{-5} \mathrm{~K}^{-n^{\prime}}$ & $n^{\prime \prime}$ & $\mathrm{K}_{n}^{\prime \prime}, 10^{-5} \mathrm{~K}^{-n}$ & $\chi^{2}, 10^{-5}$ \\
\hline \multicolumn{9}{|c|}{ Наполнитель - углеродные нанотрубки, $\omega=0,2 \%$} \\
\hline 0,0083 & 4,8 & 288 & 54 & 0,78 & 70 & 4,0 & 211 & 2 \\
\hline 0,0333 & 4,6 & 363 & 64 & 0,81 & 33 & 3,8 & 447 & 5 \\
\hline 0,0833 & 4,5 & 209 & 67 & 0,83 & 13 & 3,9 & 323 & 2 \\
\hline 0,3333 & 4,4 & 129 & 66 & 0,86 & 49 & 3,8 & 142 & 9 \\
\hline \multicolumn{9}{|c|}{ Наполнитель - углеродные нанотрубки, $\omega=0,3 \%$} \\
\hline 0,0083 & 4,4 & 440 & 86 & 0,81 & 75 & 2,20 & 854 & 2 \\
\hline 0,0333 & 4,2 & 270 & 93 & 0,85 & 39 & 2,30 & 322 & 2 \\
\hline 0,0833 & 4,1 & 250 & 95 & 0,79 & 11 & 2,30 & 180 & 1 \\
\hline 0,3333 & 4,0 & 215 & 82 & 0,75 & 21 & 2,10 & 129 & 4 \\
\hline \multicolumn{9}{|c|}{ Наполнитель - микрочастицы алюминия, $\omega=0,2 \%$} \\
\hline 0,0083 & 4,8 & 171 & 55 & 0,78 & 56 & 4,6 & 275 & 2 \\
\hline 0,0333 & 4,6 & 154 & 65 & 0,82 & 30 & 4,3 & 164 & 5 \\
\hline 0,0833 & 4,5 & 197 & 62 & 0,86 & 22 & 4,2 & 110 & 1 \\
\hline 0,3333 & 4,4 & 200 & 60 & 0,82 & 64 & 4,2 & 121 & 9 \\
\hline \multicolumn{9}{|c|}{ Наполнитель - микрочастицы алюминия, $\omega=1$ \% } \\
\hline 0,0083 & 4,6 & 150 & 98 & 0,41 & 35 & 1,2 & 176 & 7 \\
\hline 0,0333 & 4,6 & 177 & 69 & 0,85 & 39 & 1,3 & 186 & 5 \\
\hline 0,0833 & 4,5 & 315 & 93 & 0,88 & 26 & 1,1 & 341 & 4 \\
\hline 0,3333 & 4,4 & 350 & 118 & 0,79 & 42 & 1,2 & 520 & 5 \\
\hline
\end{tabular}

\section{Выводы}

1. Выполнены экспериментально-теоретические исследования по установлению механизмов структурообразования при кристаллизации из расплава полимерных композитов на основе поликарбоната, наполненных углеродными нанотрубками или микрочастицами алюминия. Исследования проведены при изменении массовой доли наполнителя $\omega$ от $0,2 \%$ до $4 \%$ и варьировании скорости охлаждения $V_{t}$ композита из расплава от 0,0083 до $0,333 \mathrm{~K} / \mathrm{c}$.

2. Экспериментально получены экзотермы кристаллизации для рассматриваемых полимерных микро- и нанокомпозитов. Выявлены эффекты влияния скорости охлаждения композита из расплава $V_{t}$ и доли наполнителя $\omega$ на различ- ные характеристики процесса кристаллизации. В частности показано, что с ростом $V$ происходит снижение максимума отводимого от композита теплового потока $Q_{\max }$ и уменьшение температур начала $T_{N}$ и конца $T_{\text {к }}$ кристаллизации при заметном повышении интервала температур кристаллизации $\Delta T$. Установлен также факт трансформации унимодального пика на экзотермах кристаллизации в бимодальный при повышении массовой доли наполнителя $\omega$.

3. С использованием результатов экспериментальных исследований в соответствии с уравнением нуклеации установлены закономерности структурообразования изучаемых композитов на начальной стадии их кристаллизации - стадии нуклеации. Показано наличие на данной стадии двух механизмов кристаллизации - плоскостно- 
го и объемного при некотором преобладании последнего.

4. С целью выявления особенностей структурообразования на второй стадии кристаллизации полимерных композитов (кристаллизация в объеме композита в целом) выполнен анализ полученных результатов экспериментальных исследований в рамках стандартного и модифицированного уравнений Колмогорова-Аврами. Установлено, что на данной стадии кристаллизация происходит по двум механизмам, первый из которых связан с кристаллизацией собственно полимерной матрицы (на флуктуациях плотности полимера), второй - с кристаллизацией, центрами которой служат частички наполнителя. Показано, что второй из указанных механизмов с увеличением содержания наполнителя трансформируется от механизма напряженной матрицы следующим образом: для композитов, наполненных углеродными нанотрубками, к двумерному поверхностному механизму, а для композитов, наполненных частицами алюминия, - к одномерному механизму образования игольчатых структур.

\section{ЛИТЕРАТУРА}

1. Hernandez-Montelongo J. Porous siliconcyclodextrin based polymer composites for drug delivery applications / J. Hernandez-Montelongo, N. Naveas, S. Degoutin, N. Tabary, F. Chai, V. Spampinato, G. Ceccone, F. Rossi, V. TorresCosta, M. Manso-Silvan, B. Martel. - Carbohydrate Polymers. - 2014. - Vol. 110. - P. 238 - 252.

2. Diaz-Bleis D. Thermal characterization of magnetically aligned carbonyl iron/agar composites [Text] / D. Diaz-Bleis, C. Vales-Pinzón, Y. FreilePelegrín, J.J. Alvarado-Gil. - Carbohydrate Polymers. - 2014. - Vol. 99. - P. 84 - 90.

3. Samanvaya S. Polymer-Particle Composites: Phase Stability and Applications in Electrochemical Energy Storage / Samanvaya Srivastava, Jennifer L. Schaefer, Zichao Yang, Zhengyuan Tu and Lynden A. Archer. - Advansed Material. - 2014. Vol. 26. - P. $201-234$.

4. Kingshuk D. Utilization of Conducting Polymers in Fabricating Polymer Electrolyte Membranes for Application in Direct Methanol Fuel Cells / Kingshuk Dutta, Piyush Kumar, Suparna Das and Patit P. Kundu. - Polymer Reviews. - 2014. Vol. 54. - P. $1-32$.

5. Parvathalu $K$. Studies of Electrical and Thermal Conductivities of Sheared Multi-Walled Carbon Nanotube with Isotactic Polypropylene Polymer Composites / Parvathalu Kalakonda, Yanial Cabrera, Robert Judith, Georgi Y. Georgiev, Peggy Cebe and Germano S. Iannacchione. - Nanomaterials and Nanotechnology. - 2015. - Vol. 5. - P. 1 - 7 .

6. Берштейн B.A. Дифференциальная сканирующая калориметрия в физикохимии полимеров / Берштейн В.А., Егоров В.М.. - Л.: Химия, 1990. - 256 c.

7. Вундерлих Б. Физика макромолекул. Т.2: Зарождение, рост и отжиг кристаллов / Вундерлих Б.. - М.: Мир, 1979. - 574 с.

8. Долинский A.A. Теплофизические свойства полимерных микро- и нанокомпозитов на основе поликарбоната / А.А. Долинский, Н.М. Фиалко, Р.В. Динжос, Р.А. Навродская // Промышленная теплотехника. - 2015. - №2. - С. 12 - 19. 


\section{STRUCTURE FORMATION OF POLYMER MICRO- AND NANOCOMPOSITES BASED ON POLYCARBONATE IN THE PROCESS OF THEIR CRYSTALLIZATION}

\section{Dolinskiy A.A. ${ }^{1}$, Fialko N.M. ${ }^{1}$, Dinzhos R.V. ${ }^{2}$, Navrodskaya R.A. ${ }^{1}$}

${ }^{1}$ Institute of Engineering Thermophysics, National Academy of Sciences of Ukraine, 2a, Zhelyabova str., Kyiv, 03680, Ukraine

${ }^{2}$ Nikolaev National University. named after V.A. Sukhomlinskiy, 24, Nikolska str., Mykolaev, 540030, Ukraine

The data of experimental and theoretical studies of the mechanisms of structure formation in the crystallization of polymer composites based on polycarbonate filled with carbon nanotubes or microparticles of aluminum are given. The effects of the influence on the performance of crystallization exotherms of the composite cooling rate from melt and the of filler share are considered. The results of the analysis of the experimental data on the crystallization kinetics in accordance with the equations of nucleation and Kolmogorov-Avrami are submitted. The mechanisms of crystallization of considered polymeric micro- and nanocomposites on the initial crystallization step (nucleation step) and the step of crystallization in the volume of material as a whole are established.

References 8, tables 4, figures 3 .

Key words: polymer micro- and nanocomposites, structure formation mechanisms, crystallization kinetics.

1. Hernandez-Montelongo J., Naveas $N$., Degoutin S., Tabary N., Chai F., Spampinato V., Ceccone G., Rossi F., Torres-Costa V., Manso-Silvan M., Martel B. Porous silicon-cyclodextrin based polymer composites for drug delivery applications // Carbohydrate Polymers. - 2014. - Vol. 110. - P. $238-252$.

2.Diaz-Bleis D., Vales-Pinzón C., Freile-Pelegrín $Y$., Alvarado-Gil J. Thermal characterization of magnetically aligned carbonyl iron/agar composites // Carbohydrate Polymers. - 2014. - Vol. 99. - P. 84 -90 .

3. Samanvaya S., Jennifer L. Schaefer, Zichao Yang, Zhengyuan Tu and Lynden A. Archer. Polymer-Particle Composites: Phase Stability and Applications in Electrochemical Energy Storage // Advanced Material. - 2014. - Vol. 26. - P. 201 - 234.

4. Kingshuk D., Piyush Kumar, Suparna Das and Patit P. Kundu. Utilization of Conducting Polymers in Fabricating Polymer Electrolyte Membranes for Application in Direct Methanol Fuel Cells // Polymer Reviews. - 2014. - Vol. 54. - P. 1 - 32.

5. Parvathalu K., Yanial Cabrera, Robert Judith, Georgi Y. Georgiev, Peggy Cebe and Germano S. Iannacchione. Studies of Electrical and Thermal Conductivities of Sheared Multi-Walled Carbon Nanotube with Isotactic Polypropylene Polymer Composites// Nanomaterials and Nanotechnology. 2015. - Vol. 5. - P. 1 - 7.

6. Bershtein V.A. Differential scanning calorimetry in the physical chemistry of polymers. L .: Chemistry, 1990. - 256 p. (Rus)

7. Wunderlich B. Physics of macromolecules. Vol.2: Nucleation, growth and annealing of crystals. - M .: Mir, 1979. - 574 p. (Rus)

8. Dolinskyi A.A., Fialko N.M., Dinzhos R.V., Navrodskaya R.A. Thermophysical properties of polymer micro- and nanocomposites based on polycarbonate // Industrial Heat Engineering. - 2015. - № 2. - P. 12 - 19. (Rus) 